\title{
Interference in memory for tonal pitch: Implications for a working-memory model
}

\author{
THOMAS PECHMANN and GILBERT MOHR \\ Universität des Saarlandes, Saarbrücken, Germany
}

\begin{abstract}
The degree of interference caused by different kinds of stimuli on memory for tonal pitch was studied. Musically trained and untrained subjects heard a sequence of two tones separated by an interval of $5 \mathrm{sec}$. The tones were either identical in pitch or differed by a semitone. Subjects had to decide whether the tones were identical or not. The interval was filled with tonal, verbal, or visual material under attended and unattended conditions. The results revealed clear group differences. Musically trained subjects' retention of the first test tone was only affected by the interposition of other tones. In contrast, the performance of musically untrained subjects was also affected by verbal and visual items. The findings are discussed in the framework of Baddeley's (1986) working-memory model.
\end{abstract}

In a number of experiments, Deutsch studied the effect of short sequences of tones on the retention of tonal pitch (see Deutsch, 1982, for an overview). These experiments principally employed the same method, which is illustrated in Figure 1. Subjects heard a first test tone followed by a series of six interpolated tones varying in pitch. All tones had a duration of $200 \mathrm{msec}$ and were separated by short pauses of $300 \mathrm{msec}$. At the end, there was a longer pause of $2,000 \mathrm{msec}$ duration and then a second test tone was presented that was either identical in pitch with the first test tone or not. The subjects were asked to compare the pitches of the first and second test tones. The dependent variable was the number of errors. Deutsch mainly varied the frequency and the position of a critical interpolated tone as independent variables, holding the timing skeleton and the number of intervening tones constant. She found maximal interference if the critical tone was the second one in the series of intervening tones and if it differed from the initial test tone by two thirds of a tone. In this condition, subjects made $51 \%$ errors if the two test tones were different and $21 \%$ if they were identical (Deutsch, 1975).

Deutsch explained this effect in terms of lateral inhibition and provided additional evidence for this claim by showing disinhibitory effects on the first test tone by inhibition of the second interpolated tone (Deutsch, 1984;

\footnotetext{
This is a revised version of a paper presented at the Fourth Conference of the European Society for Cognitive Psychology at Como, Italy, in September 1990. Both authors contributed equally to this research. We are grateful to Bernhard Morsch for collecting part of the data. Thanks are also due to Herb Clark, Johannes Engelkamp, Pim Levelt, Antje Meyer, Piet Vos, and Pienie Zwitserlood, who commented on an earlier draft of this paper. In addition, we would like to thank Robert Crowder, Robert Proctor, and two anonymous reviewers for helpful suggestions concerning revision. Correspondence should be addressed to T. Pechmann, Universität des Saarlandes, Fachrichtung Psychologie, D-6600 Saarbrücken, Germany.
}

Deutsch \& Feroe, 1975). The explanation based on lateral inhibition might be further specified by reference to the concept of the critical bandwidth (cf. Plomp \& Levelt, 1965). Since the size of the critical band depends on its center frequency, the distance between two tones laterally inhibiting each other might depend on the range of frequencies being investigated. However, this issue has not been taken up in the literature.

If lateral inhibition is the crucial mechanism, the most efficient pitch eraser should consist of tonal material. But what about speech or other acoustic stimuli? Do they have a detrimental effect as well, and is it within the same range? In one of her earlier experiments, Deutsch (1970) studied the effects of interpolated tones and spoken.numbers on memory for tonal pitch using her standard paradigm (described above). Her results displayed a detrimental effect of tonal items but no effect of interpolated speech. She concluded that to obtain interference effects on memory for pitch, one has to use highly specific material.

Lateral inhibition of tonal pitch is a function of both frequency and temporal distance. In a number of experiments, Deutsch also varied the serial position of the critical interpolated tone and found that it produced substantially more interference when it was included early rather than late in the sequence. Thus, if the memory for tonal pitch is only disrupted by lateral inhibition, its representation should be firm and safe against any attempt of further interference once it has survived the stage sensitive to lateral inhibition.

So far, we might assume that memory for pitch in a recognition task like that designed by Deutsch is independent of the musical expertise of individual subjects, since the mechanism of lateral inhibition can hardly be influenced by training. But what about later stages? In Deutsch's experiments, the tonal representation had to somehow be maintained until the second test tone was presented. Does pitch maintenance during this retention phase re- 


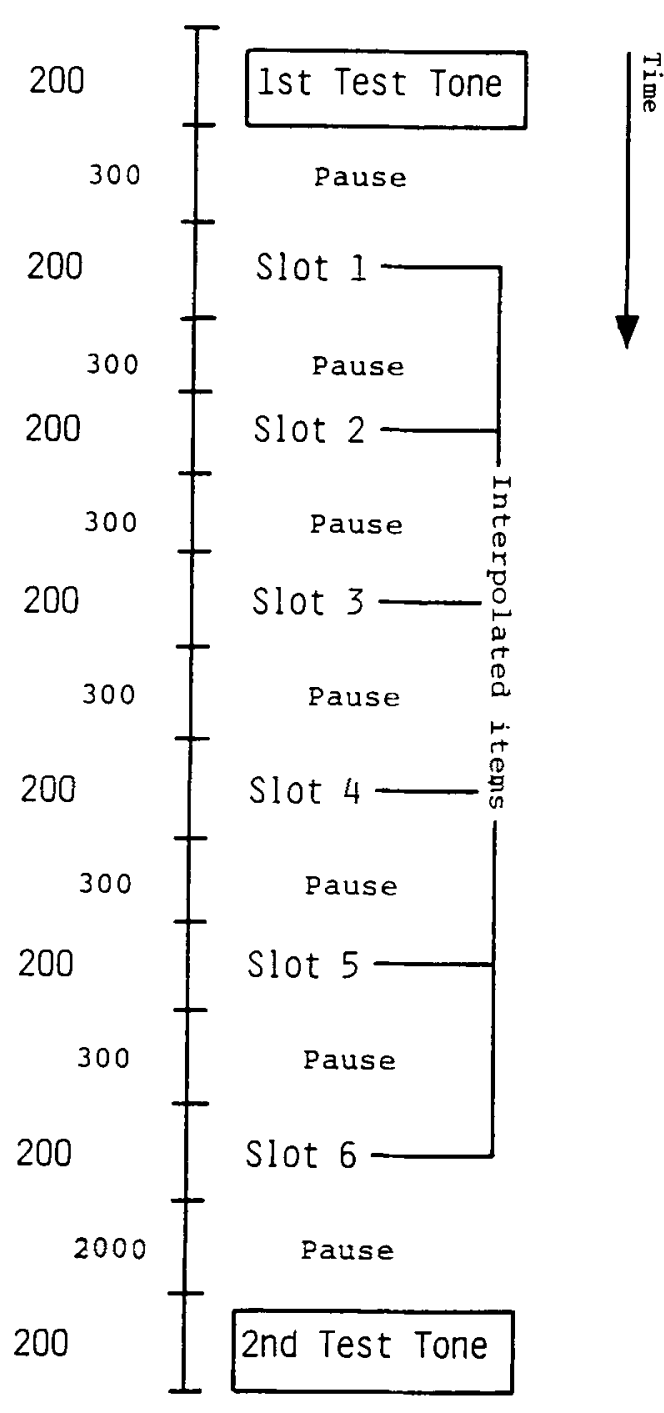

Figure 1. Timing skeleton used in Deutsch's experiments (see Deutsch, 1975).

quire a substantial amount of attention? If the answer is positive, does subjects' experience with musical material play any role?

In a study in which spoken numbers were used as interpolated stimuli, Deutsch (1970) also included a condition in which the subjects had to recall the list of numbers. But even in this condition, the spoken words did not affect subjects' memory for tonal pitch. Thus, one might conclude that the maintenance of tonal pitch does not require any substantial amount of central capacity. However, we do not know whether Deutsch used musically trained or untrained subjects, yet this factor might play a significant role. It is conceivable that musically trained subjects differ from musically untrained subjects in the amount of attention required to maintain a tonal representation over time and under different conditions.
This claim might be substantiated by reference to the working-memory model proposed by Baddeley (1986). This model is characterized by a device called the central executive and a number of slave systems. The central executive is responsible for monitoring and controlling the smooth functioning of the slave systems and for distributing attentional resources if necessary. The function of the slave systems is to preserve the incoming perceptual information as long as necessary for the task in which the subject is involved. Two such slave systems have been studied in detail by Baddeley and co-workers: the visuospatial sketchpad and the articulatory loop. They are designed as active devices: to keep the information in store, it must be regularly refreshed. In the case of the visual system, it is speculated that eye movements fulfill this function. In the case of the auditory system, the proposed mechanism is subvocal rehearsal.

The articulatory loop is designed to process linguistic material. It consists of a phonological store and an articulatory refreshing mechanism. The assumption of the latter is supported, for instance, by experiments demonstrating that suppressing the (subvocal) articulation of tobe-learned vertal material by a concurrent (overt) articulatory task affects the retention of the material (Murray, 1967).

One might assume that musical pitch is retained by a very similar mechanism: a kind of "tonal" loop. If subjects are asked to retain the pitch of a particular tone for some time, they usually report afterwards that they internally rehearsed the tone. However, the efficiency and automaticity of a tonal loop may be crucially dependent on subjects' musical training. The articulatory loop is assumed to work rather automatically, that is, without the requirement of central resources. In contrast, at least for musically untrained people, tonal rehearsal may be a much more controlled process that requires additional allocation of attention. Thus, musical training may significantly affect the functioning of the loop.

With respect to the processing of speech and music in working memory, the additional question arises whether they are processed in a common acoustic system or in two different, specialized systems. The basic line of argumentation underlying memory research and the workingmemory model in particular is that if the working of a system is not affected by the concurrent processing of a particular kind of material, then this material is processed in another system. According to this argument, the findings obtained by Deutsch (1970) that concurrent speech does not affect the retention of tonal pitch suggest that music and speech are processed in two different memory systems. But then the opposite should also be true: the processing of linguistic items should not be affected by the concurrent processing of musical stimuli.

This issue has been taken up in a recent study by Salame and Baddeley (1989). They investigated the effects of concurrent speech, noise, or music on the retention of visually presented verbal material. They found that noise had no 
detrimental effect. Music, however, significantly disrupted short-term memory performance compared with silence, although it was less disruptive than speech. For subjects who had previously performed a separate memory experiment for an hour, only vocal music interfered with memory performance. For less-prepared subjects, both vocal and instrumental music had a detrimental effect.

Salamé and Baddeley discuss these results in the light of a so-called phonological-detector hypothesis. The core meaning of this notion is that "the short-term storage component of the articulatory loop system is not a general acoustic store protected by a filter, but is rather a detection and storage system that has evolved for processing speech-like material"' (Salamé \& Baddeley, 1989, p. 121). This hypothesis might explain why concurrent vocal music has a stronger disruptive effect on the retention of speech than does instrumental music. But why does instrumental music interfere with speech at all? Salamé and Baddeley concede that "the fact that we can hear and remember sounds that are very unlike speech means that there must be some-presumably additional-form of acoustic storage system capable of dealing with such material" (Salamé \& Baddeley, 1989, p. 121).

The results obtained by Salamé and Baddeley (1989) suggest that speech and music are processed in a common system of working memory. The contradiction is obvious: the experiments performed by Deutsch (1970) showed that concurrent speech did not affect tonal memory, suggesting that speech and music are processed in two different systems.

The experimental work reported in the present paper was done in an attempt to clarify some of the issues discussed above. This was done by studying the effects of different classes of interpolated stimuli on subjects' memory for tonal pitch using the methodological paradigm developed by Deutsch. Our first concern was to replicate her finding (Deutsch, 1970) that tonal memory is not affected by concurrent speech, which in a certain sense is at odds with the results obtained by Salamé and Baddeley (1989). Second, we were interested in learning whether the maintenance of tonal pitch depends on attentional resources. Third, we addressed the question of whether subjects' memory for pitch while processing other material depends on their musical expertise. Finally, we also investigated whether tonal memory is affected by the concurrent processing of nonacoustic, visual stimuli under both attended and unattended conditions.

\section{EXPERIMENT 1}

\section{Method}

Subjects. Twenty-seven students at the University of Saarbrücken volunteered as subjects. Fourteen of them were trained musicians; 13 were musically untrained. The criterion of being musically trained was a rather unrefined one. The subjects were classified as belonging to this group if they actively played an instrument. All of the musicians were amateurs. None of the subjects possessed absolute pitch.
Materials and Procedure. We used the experimental procedure developed by Deutsch as a timing skeleton for all conditions, filling the slots for interpolated items with different classes of stimuli. Our experiment included the following conditions.

First, a control condition was developed in which we simply presented the two test tones at an interval of $5 \mathrm{sec}$ without any interfering material. In the second condition, the slots for interfering items were filled by a sequence of tones following Deutsch's procedure. The subjects were instructed to concentrate only on the two test tones. Then two verbal conditions were included. In the third condition, the slots were filled by monosyllabic words that the subjects again were instructed to ignore (unattended condition). The fourth condition was identical with the third condition except that the subjects had to do a secondary task concerning the verbal stimuli (attended condition). Finally, two visual conditions were developed. In the fifth condition, visual stimuli were presented as interfering items that the subjects were supposed to ignore, but in the sixth condition these stimuli were used for a secondary task.

Under all conditions, the subjects first received an attention cue that was a tone of $4000 \mathrm{~Hz}$ presented for $50 \mathrm{msec}$. It was followed by a pause of $950 \mathrm{msec}$. Then the first test tone, with a duration of $200 \mathrm{msec}$, was presented. It was followed by a pause of $300 \mathrm{msec}$, at the end of which the series of intervening items began.

Each intervening item had a duration of $200 \mathrm{msec}$ and was followed by a pause of $300 \mathrm{msec}$. After the sixth and last intervening item, the pause extended to $2,000 \mathrm{msec}$. Then the second test tone was presented for $200 \mathrm{msec}$, followed by a pause of $4,000 \mathrm{msec}$. During this last pause, the subject's verbal reaction ("same"/."different") was expected. After this 4,000-msec interval, the next trial started.

The whole experiment was run under the control of a personal computer that also generated the tones presented via earphones. The subjects received 48 items under each condition. In the control condition, the subjects heard the first and second test tones with nothing in between. In half of the cases, the two test tones were identical; in half of the cases they differed by a semitone, with an equal number of tones being higher or lower.

The test tones were taken from the 12 semitones of one, octave ranging from 262 to $494 \mathrm{~Hz}$. Every semitone was used four times as the first member of a pair of test tones. In two of these four pairs, the tones were identical. In one of the remaining two pairs, the second tone was higher; in the other pair it was lower. Three different pseudorandomized lists of these 48 items were prepared under the restriction that identical first test tones could only reoccur after two other items. These three lists were used both in ascending and descending order, which actually made six lists in all. Each of these six lists was assigned to one of the six conditions. The sequence of conditions was systematically varied across subjects.

The tonal condition was identical to the control condition except that intervening tones were included following Deutsch's procedure. The intervening tones consisted of the 24 semitones of two octaves ranging from 185 to $698 \mathrm{~Hz}$. The second intervening tone always differed from the first test tone by two thirds of a tone. The other intervening tones were a random sample of the 24 semitones of the two octaves we were using, under the following restrictions. First, neither of the two test tones accurred in the sequence of interpolated tones. Second, if the second test tone was higher in pitch than the first one, the second interpolated tone was lower, and vice versa.

In the two verbal conditions, the six intervening tones were replaced by six monosyllabic words spoken by a male voice. We used nouns only. In half of the cases, the last two words rhymed; in the other half they did not. The items had been tape-recorded. In the unattended verbal condition, the subjects heard the words but were only asked to judge the two test tones. In the attended condition, the subjects were asked to indicate by pressing one of two buttons whether the last two words rhymed before they received the second test tone, which they then had to compare with the first 
test tone. Two series of words were prepared that were alternately assigned to the unattended and attended conditions across subjects.

In the visual conditions, the slots for the intervening stimuli were occupied by $4 \times 4$ matrices (Figure 2 ) presented on a computer screen following a procedure used by Phillips (1974). In each case, 5 of the 16 cells of each matrix were filled. The position of the filled cells varied at random for each matrix, but in half of the trials the last two matrices were identical. Again, two visual conditions were performed. In the unattended condition, the subjects were asked to judge only whether the two test tones were identical. In the attended condition, they were additionally asked to indicate by pressing buttons whether the last two matrices were identical. They were instructed to respond directly after they had seen the last matrix.

The whole experiment lasted about $1 \mathrm{~h}$.

\section{Results}

A three-way analysis of variance was calculated with conditions, groups (musicians and nonmusicians), and identicalness of test tones as factors. A significant effect of conditions was found $\left[F(5,125)=201.02, M S_{c}=\right.$ $4.63, p<.001]$. A post hoc Scheffe test showed that the tonal condition significantly differed from all other conditions. In addition, the verbal-attended condition differed from the visual-unattended and the control conditions. No further differences were statistically significant $\left(\right.$ Diff $\left._{\text {crit(Scheffe }) / p}=.05=3.02\right)$.

The groups factor was also significant $[F(1,25)=$ $\left.25.49, M S_{e}=10.3, p<.001\right]$. Musicians made significantly fewer errors than did nonmusicians, $4.17(9 \%)$ compared with 7.78 (16\%). The main effect of groups was qualified by a significant interaction between conditions and groups $\left[F(5,125)=5.10, M S_{c}=4.63, p<\right.$ $.001]$. Table 1 shows the corresponding mean errors for the two groups and the six conditions. A Scheffé test

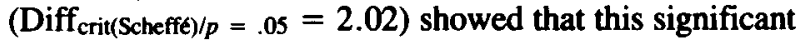
interaction can be attributed to the fact that for the musicians, interference was found only in the tonal condition. In all other conditions, no interference effect was observed for this group. For nonmusicians, in contrast, the analysis not only yielded significant differences between the tonal condition and all other conditions, but also between the verbal-attended condition and all other conditions. Furthermore, the verbal-unattended and the visual-attended conditions differed significantly from the visual-unattended and the control conditions.

The factor identicalness of test tones was also significant $\left[F(1,25)=59.41, M S_{\mathrm{e}}=7.26, p<.001\right]$. Generally, the subjects made more errors when the two test tones were different $(4.09$, or $17 \%)$ than when they were the same $(1.81$, or $8 \%)$, but this effect was particularly strong in the tonal condition and in the verbal-attended condition as mirrored by the significant interaction between the factors identicalness of test tones and conditions $[F(5,125)=$

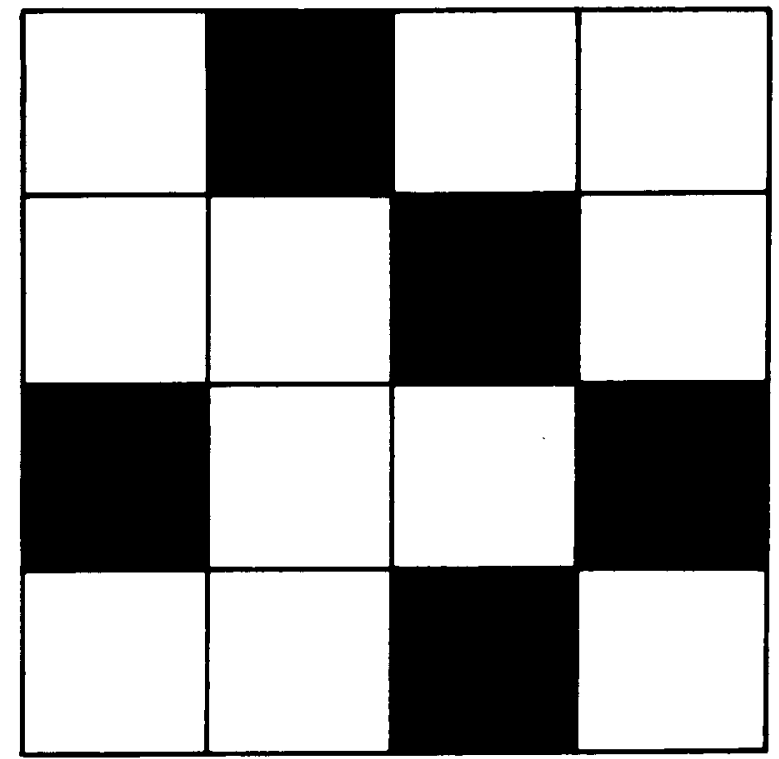

Figure 2. Example of visual stimuli used in Experiment 1 (adapted from Phillips, 1974).

28.98, $\left.M S_{\mathrm{e}}=4.89, p<.001\right]$ (Table 2). According to a Scheffé post hoc test $($ Diff $\operatorname{crit}($ Scheffe $) / p=.05=2.04)$, the differences between identical and nonidentical test tones were statistically significant only in the tonal and the verbal-attended conditions.

The interaction between groups and identicalness of test tones was also significant $\left[F(1,25)=5.31, M S_{c}=\right.$ 7.26, $p=.03$ ] (Table 3). A corresponding Scheffé test $\left(\right.$ Diff $\left._{\mathrm{crit}(\text { Scheffe }) / p}=.05=2.18\right)$ revealed a significant difference between identical and nonidentical tones for nonmusicians only. Furthermore, nonmusicians made a higher number of errors than did musicians in the nonidentical condition, but not in the identical condition. The three-way interaction was nonsignificant $[F(5,125)=1.58, p=.17]$.

Finally, we inspected the number of errors in both of the attended secondary tasks. The effect of secondary tasks on a primary task might depend solely on the difficulty of the secondary tasks as such. Indeed, we found that the subjects made significantly more errors in the visual secondary task $(21 \%)$ than in the verbal secondary task (1.5\%). Thus, one might argue that we must take into account a different tradeoff concerning the subjects' attention directed to the primary and secondary tasks with respect to the visual and verbal conditions. If the subjects paid much less attention to the visual secondary task than to the verbal task, this might explain why the visual task did not interfere with the retention of the test tones. How-

Table 1

Mean Number of Errors Under the Six Conditions for the Two Groups of Subjects

\begin{tabular}{lcccccc}
\hline & Control & $\begin{array}{c}\text { Visual/ } \\
\text { Unattended }\end{array}$ & $\begin{array}{c}\text { Visual/ } \\
\text { Attended }\end{array}$ & $\begin{array}{c}\text { Verbal/ } \\
\text { Unattended }\end{array}$ & $\begin{array}{c}\text { Verbal/ } \\
\text { Attended }\end{array}$ & Tonal \\
\hline Musicians & 0.21 & 0.14 & 0.14 & 0.36 & 1.64 & 22.5 \\
Nonmusicians & 1.92 & 2.54 & 4.85 & 5.31 & 9.23 & 22.8 \\
\hline
\end{tabular}


Table 2

\begin{tabular}{lcccccr}
\multicolumn{6}{c}{ Mean Number of Errors as a Function of Condition and Identicalness of Test Tones } \\
\hline & Control & $\begin{array}{c}\text { Visual/ } \\
\text { Unattended }\end{array}$ & $\begin{array}{c}\text { Visual/ } \\
\text { Attended }\end{array}$ & $\begin{array}{c}\text { Verbal/ } \\
\text { Unattended }\end{array}$ & $\begin{array}{c}\text { Verbal/ } \\
\text { Attended }\end{array}$ & Tonal \\
\hline Identical & 0.41 & 0.26 & 0.89 & 0.89 & 1.48 & 6.93 \\
Nonidentical & 0.63 & 1.04 & 1.52 & 1.85 & 3.82 & 15.70 \\
\hline
\end{tabular}

ever, this argument is made less plausible by the findings of Experiment 2, in which the subjects were given only two secondary tasks.

\section{EXPERIMENT 2}

\section{Method}

Subjects. Ten new subjects participated in the experiment. All of them were students of the University at Saarbrücken.

Materials and Procedure. The subjects were presented the two secondary tasks prepared for Experiment 1, using the identical material and timing skeleton. Half of the subjects started with the visual task; the others started with the verbal task.

\section{Results and Discussion}

The results were clear-cut. The subjects made simply no errors at all in the verbal condition, but $11 \%$ errors in the visual condition. All the subjects but one contributed to the errors in this condition. The difference between the two conditions is statistically highly significant $[t(9)=$ $3.86, p<.01]$.

We concluded that the different degree of interference by our verbal- and visual-attended tasks on the retention of musical tones by untrained subjects in Experiment 1 cannot be attributed to the difference in difficulty of the two tasks. Instead, the verbal task, which was clearly less difficult than the visual task, caused a much greater number of errors in the primary task. Consequently, the nature of the material, but not the general difficulty of the secondary task, was responsible for the number of errors made by the nonmusicians in different conditions.

\section{GENERAL DISCUSSION}

The resuits can be easily summarized. First, if the subjects were musically trained, only the tonal condition significantly interfered with the retention of the first test tone. Second, for the subjects who were not trained, memory of the first test tone was also affected by the two verbal conditions and by the visual-attended condition, although the number of errors in these conditions was still substantially smaller than in the tonal condition. Third, there was a greater tendency for the subjects to erroneously hear no difference between two nonidentical tones than to er-

Table 3

Mean Number of Errors as a Function of Groups and Identicalness of Test Tones

\begin{tabular}{lcc}
\hline & Musicians & Nonmusicians \\
\hline Identical & 1.26 & 2.89 \\
Nonidentical & 2.39 & 5.19 \\
\hline
\end{tabular}

roneously hear a difference between two identical tones. This effect was strongest under the two most difficult conditions, the tonal and the verbal-attended conditions.

We would like to discuss three points. First, we will distinguish two types of interference. Second, the difference between identical and nonidentical test stimuli will be discussed. Finally, we will consider the implications concerning the group differences we obtained.

Two aspects of our results support the assumption that two fundamentally different types of interference were observed. First, our data revealed a sharp decline in the number of errors from the tonal condition to the other interference conditions. The subjects' performance in the tonal condition was at chance level, whereas the other conditions caused either no interference at all or only weak interference. It is unlikely that this difference can be explained by a particularly large degree of attention that the subjects allocated to the interfering stimuli in the tonal condition, since they had been explicitly instructed to ignore them. Second, the data showed a clear interaction between musical expertise and interference conditions. Musical training did not prove to have any effect on performance in the tonal condition, but it substantially changed the picture in all other conditions. We conclude that in the tonal condition, data-limited processes in the sense of Norman and Bobrow (1975) were responsible for the subjects' failure to reliably retain the first test tone: the subjects' performance could be improved neither by individual expertise nor by maximal allocation of central resources. The results suggest that tonal interference was due to mechanisms that might be identified with lateral inhibition, as proposed by Deutsch. We suggest speaking of peripheral interference in this case. Here, the term peripheral should not be understood in the sense of an early sensory storage mechanism that is, for instance, open to masking effects (cf. Cowan, 1984). Rather, we want to refer by this term to a nonsensory storage mechanism that is susceptible to overwriting by new sensory input and that is hardly controlled by central processes (cf. Phillips, 1974, for a corresponding proposal concerning the processing of visuospatial information).

In contrast to the tonal condition, performance in all other interference conditions proved to be susceptible to the allocation of attentional resources and to the subjects' musical expertise. Musicians were obviously able to run tone-maintenance processes rather efficiently in these conditions. Neither irrelevant stimuli nor attention-demanding secondary tasks had any effect on their performance. In contrast, the performance of the nonmusicians was significantly dependent on both the availability of attentional 
resources and on the degree of similarity between the target and interfering stimuli. The impact of attentional resources becomes particularly evident regarding the visual stimuli: although they were most dissimilar from the target stimuli and did not interfere in the unattended condition, they caused significant interference in the attended condition. The impact of the mere similarity between target and interfering stimuli is particularly evidenced by the unattended verbal condition: acoustically presented speech interfered with tonal memory even when no additional attention was required. The implication is twofold. First, one should distinguish interference effects caused by stimulus quality from effects caused by task requirements. Second, one should bear in mind that different stimuli may have effects on different stages of information processing. Consequently, one should be rather careful in interpreting interference effects of particular stimuli without considering and testing the locus of the effect.

The second point we want to discuss has to do with the identicalness factor. Even in the tonal condition, which we assume to produce peripheral interference, subjects performed significantly better with identical than with nonidentical tones, the performance with identical tones being above chance level. This might suggest that the identical decisions were based on different processes than were the nonidentical decisions. The processes relevant for the nonidentical decisions were apparently more susceptible to peripheral interference than were the processes relevant for the identical decisions.

This difference might be explained as follows. In both cases, the subject had to retrace the representation of the first test tone when the second one was presented. If the representation of the first test tone had been blurred by the interfering stimuli, it might then have been comparatively easy to retrieve a former memory trace when it was refreshed by an identical copy triggered by the repeated presentation of the initial stimulus as compared with the presentation of a different stimulus. In other words, the repetition of the identical tone might have resonated with the initial tone, reactivating the blurred memory trace.

The subject of our third point is the different performance of musicians and nonmusicians. First, one might suspect that these differences did not originate in the subjects' expertise but in different decision criteria. According to this hypothesis, nonmusicians had a more liberal response criterion (a tendency to respond "same"), while the musicians responded more conservatively.

The possibility of calculating indices for a response bias turned out to be restricted by our data. First, the musicians' performance was almost perfect in all conditions but the tonal condition. Consequently, it only makes sense to calculate a response-bias index for the tonal condition. Second, in all other conditions, the performance of the nonmusicians was also almost perfect within the identical trials. A calculation of the bias index $\mathrm{Br}$ [false alarms $/ 1$ - (hits - false alarms)] for the tonal condition (see Snodgrass \& Corwin, 1988) yielded no group effect. Both groups had a slightly liberal bias. The Br score for musicians was .73; the score for nonmusicians was .69 (the score ranges from liberal $=1.0$ to conservative $=0.0$ ).

Regarding the performance of the nonmusicians confronted with nonidentical trials in the other conditions, inspection of the data clearly showed different rates of false alarms across conditions. This finding makes the assumption of a particular bias of this group rather unlikely. In summary, we conclude that the differences between the musicians and the nonmusicians were due to expertise and not to response criteria.

But how, then, can the differences in performance between the two groups be explained? We suggest that they were due to a different efficacy of a tonal loop. The performance of the trained subjects was not affected by nontonal stimuli, because the tonal loop ran smoothly and pretty automatically even when the interfering stimuli demanded additional attention. In contrast, the tonal loop required a substantial amount of attention from the untrained subjects. Consequently, even the processing of nonacoustic, visual stimuli deteriorated the retention of pitch information when it was attention-demanding.

Of course, we do not claim that the superior performance of our musically trained subjects necessarily derived from their training. It might well be the case that they sought out and received musical training because they had good memories for pitch. This point raises the suggestion of using very carefully selected subjects in future research. To study more closely what it is that differentiates musicians from nonmusicians in experiments like ours, it would be necessary to understand better what the discriminating variables are between good and poor performers. Furthermore, it remains to be seen whether tonal interference is as uncontrollable a mechanism as lateral inhibition suggests or whether subjects can be found who remain unaffected.

We suggested above that the tonal loop can be conceived as a sister of the articulatory loop. Thus, we assume that it consists of a tonal-storage component and a tonal-rehearsal component. Nonmusicians' performance might be affected by nonoptimal processing in both components. Our data do not provide for the possibility of assigning their failure to one or the other of these components.

Alternatively, working memory might consist of one common acoustic store in which both speech and tonal information are processed, supplemented by specialized rehearsal mechanisms. Again, we do not feel that a decision can be made concerning the architecture of the corresponding part of working memory on the basis of the available empirical evidence.

However, irrespective of a decision between the alternatives, our findings seem to strongly point to the value of conceiving of working memory not as a static structure that is interindividually constant, but as being dependent on individual or group differences. This aspect seems to have been widely neglected in the past, probably because research almost exclusively concentrated on ver- 
bal and visual behavior from which the necessity of taking interindividual or group differences into account is less clear.

\section{REFERENCES}

BADDELEY, A. (1986). Working memory. Oxford: Oxford University Press.

Cownn, N. (1984). On short and long auditory stores. Psychological Bulletin, 96, 341-370.

DEutsCH, D. (1970). Tones and numbers: Specificity of interference in short-term memory. Science, 168, 1604-1605.

DEUTSCH, D. (1975). The organization of short-term memory for a single acoustic attribute. In D. Deutsch \& J. A. Deutsch (Eds.), Short term memory (pp. 107-151). New York: Academic Press.

Deutsch, D. (1982). The processing of pitch combinations. In D. Deutsch (Ed.), The psychology of music (pp. 271-316). New York: Academic Press.

DeuTsCH, D. (1984). Memory for nonverbal auditory information: A link between behavioral and physiological studies. In $\mathbf{L}$. R. Squire \& N. Butters (Eds.), Neuropsychology of memory (pp. 45-54). New York: Guilford.
Deutsch, D., A Feroe, J. (1975). Disinhibition in pitch memory. Perception \& Psychophysics, 17, 320-324.

MuRRaY, D. J. (1967). The role of speech responses in short-term memory. Canadian Journal of Psychology, 21, 263-276.

Norman, D. A., Bobrow, D. G. (1975). On data-limited and resource-limited processes. Cognitive Psychology, 7, 44-64.

PHILLIPS, W. A. (1974). On the distinction between sensory storage and short-term visual memory. Perception \& Psychophysics, 16, 283-290.

Plomp, R., Levelt, W. J. M. (1965). Tonal consonance and critical bandwidth. Joumal of the Acoustical Society of America, 38, 548-560.

SALAMÉ, P., \& BADDELEY, A. (1989). Effects of background music on phonological short-term memory. Quarterly Journal of Experimental Psychology, 41A, 107-122.

Snodgrass, J. G., \& Corwin, J. (1988). Pragmatics of measuring recognition memory: Applications to dementia and amnesia. Journal of Experimental Psychology: General, 117, 34-50.

(Manuscript received May 17, 1991; revision accepted for publication October 9, 1991.)

\section{Call for Assistance in The Compilation of a History of the Psychonomic Society}

The Governing Board of the Psychonomic Society is pleased to announce that Robert C. Bolles has agreed to serve as the first Historian of the Society.

All members who might have information relevant to this undertaking are invited to send it directly to Dr. Bolles. Founding members and those who attended the early meetings are especially encouraged to record their reminiscences. While Dr. Bolles hopes to collect as much information as possible relevant to the history of the Society, he will concentrate first on the early history.

Dr. Bolles's address is Department of Psychology, University of Washington, Seattle, Washington 98195 (phone: 206-543-2631). 